\title{
Centro de Tratamento Oncológico: A situação da rede de tratamento de câncer de Araçatuba e região
}

\section{Cancer Treatment Center: The situation of network of treatment of cancer in Araçatuba and region}

Centro De Tratamiento Contra el Cáncer: La situación de la red de tratamiento contra el cáncer de Araçatuba y región.

Natalia Zorzenon Maia Graduanda, UNIP, Araçatuba, Brasil. nati.zorzenon.maia@gmail.com

Gislaine Bianchi Especialista, UNIP, Araçatuba, Brasil. gbianchi.arq@gmai.com

Ricardo Sueta Especialista, UNIP, Araçatuba, Brasil. ricardoutimura@yahoo.com 


\section{INTRODUÇÃO}

O câncer, doença gravíssima para a humanidade, representa um dos maiores índices de morte no Brasil e no Mundo. No estado de São Paulo, estima-se que, entre os anos de 2010 e 2013, a mortalidade decorrente das neoplasias cancerígenas foi de 184.293 pessoas, sendo 98.933 homens e 85.360 mulheres, conforme o levantamento feito pelo INCA (Instituto Nacional de Câncer), em 2014.

Essa doença é tratada pela área de estudo denominada Oncologia. A definição desta palavra encontrada em diversas fontes secundárias. Ora apresenta-se a encontrada no site do Centro de Combate ao Câncer (Especificada em Abril de 2016) é:

\footnotetext{
Oncologia é a especialidade médica que estuda as neoplasias, ou tumores, benignas ou malignas. A origem da palavra vem do grego onkos, que significa volume, que sabemos atualmente ser causado pelo acúmulo de células cancerosas em regiões do organismo. Os tumores podem ser localizados ou invadir outros tecidos, disseminando-se por todo o corpo.
}

Os serviços de Oncologia recebem o auxílio de outros tipos de especialidades, como exemplo: Pediatria, Radiografia, Psiquiatria, Cirurgia, dentre outros. É imprescindível o envolvimento de outras especialidades médicas para o tratamento que se pretende efetivo. Cancerologia é sinônimo de Oncologia.

Nesse contexto, a arquitetura desenvolve um grande papel, que é o de simplificar e melhorar as condições físicas do local, influenciando à melhora física do paciente também. Decorrente da atenção que vem ganhando, a arquitetura hospitalar com seu modo de atender as necessidades dos usuários, deixa de realizar um projeto simplório para consolidar um projeto humanizado e acolhedor, modificando suas características tradicionais, desde a fase do planejamento ao acabamento.

Portanto o objetivo desta pesquisa é demonstrar a atual situação da rede de tratamento de câncer em Araçatuba a qual atende 42 municípios da região.

\section{OBJETIVO}

Atualmente, devido ao o aumento de casos de câncer na região, necessita-se de um espaço que a atenda em sua totalidade, apesar de em Araçatuba, existir uma unidade para tratar casos de câncer, é importante destacar que existe uma fila de espera muito grande, a qual engloba pacientes para se consultar, até os que necessitam de início do tratamento.

Portanto, o objetivo deste trabalho é demonstrar a atual necessidade de melhoria na rede pública de tratamento de câncer em Araçatuba e região. 


\section{METODOLOGIA}

Este estudo classifica-se como exploratório porque é realizado por meio de levantamentos bibliográficos, a fim de encontrar estudos e teorias que forneçam informações para o desenvolvimento da pesquisa.

As referências contidas encontram-se nas fontes secundárias, que estão nos livros, artigos científicos impressos e ou disponíveis em websites etc.

\section{HISTÓRIA DOS HOSPITAIS MUNDIAIS}

A arquitetura hospitalar sofreu diversas transformações decorrentes dos avanços das tecnologias, das descobertas na área da saúde e da evolução do saber da sociedade.

Durante a Idade Média, os hospitais eram associados à morte. $O$ objetivo era o confinamento das pessoas doentes, visando mais a proteção dos que estavam fora dos hospitais do que o atendimento aos pacientes. Havia pouca esperança de recuperação. Segundo Miquelin (1992, apud LUCKIANTCHUKI; CARAM, 2011, p.3). Os hospitais eram ambientes insalubres, a circulação de ar era considerada impura. As janelas tinham proporções pequenas, deixavam o ambiente escuro e assustador.

Considerado um local de depósito de doentes, as pessoas, na sua maioria, não retornavam desses ambientes insalubres com vida, sendo assim denominados de Salle de Mourir (Quarto de morte). Segundo Costi (2002, apud LUCKIANTCHUKI; CARAM, 2011, p.3). Na qual os ambientes eram escuros e com pouca ventilação.

No entanto, por volta de 1780, quando a doença passa a ser reconhecida como fato patológico, o hospital se torna destinado a curar aparece claramente em torno de 1780 e é assinalada por uma nova prática: a visita e a observação sistemática e comparada dos hospitais.

Segundo Miquelin (1992, apud LUCKIANTCHUKI; CARAM, 2011, p.4), na Inglaterra, a enfermeira Florence Nightingale constituiu-se o elemento mais importante e característico da anatomia hospitalar do fim do século XIX, na virada do século XX, o modelo PavilhonarNightingale. Foi mantido como referência de arquitetura na saúde, porque possuía as seguintes características: os leitos eram encostados nas paredes, com banheiros e cozinhas bem ventiladas, pé direito menor para melhor controle da temperatura, as janelas ficavam frente uma a outra e também maiores para possibilitar melhor entrada de luz natural. A importância da ventilação e da insolação se propagou, arejando e higienizando os interiores dos hospitais, segundo Costi ( 2002, apud LUCKIANTCHUKI; CARAM, 2011, p.3).

A ventilação e a iluminação foram tendo prioridades nas construções de hospitais com a preocupação com a higiene e com o calor do sol para reduzir a contaminação do ambiente. Os hospitais passam a ser vistos como locais onde a vida pode não somente ser salva, mas ter sua qualidade melhorada. Segundo Miquelin (1992, apud LUCKIANTCHUKI; CARAM, 2011, p.6). 
Conforme OMS põe em ênfase a importância da arquitetura hospitalar, na qual pode além de ajudar na cura, possibilita aos pacientes espaços para descanso e qualidade, proporcionando o melhor bem estar do paciente não só físico, como explica a citação à baixo:

Saúde é o estado de mais completo bem-estar físico, mental e social, e não apenas a ausência de enfermidade. (Organização Mundial de Saúde - Conferência de Alma Ata, 1978).

Um instrumento destinado a curar. Segundo Foucault (1989, apud LUCKIANTCHUKI; CARAM, 2011, p.4)

O hospital como instrumento terapêutico é uma invenção relativamente nova, que data do final do século XVIII. A consciência de que o hospital pode e deve ser um instrumento

\section{HISTÓRIA DOS HOSPITAIS DO BRASIL}

As primeiras Santas Casas do Brasil chegaram juntamente com os padres da Companhia de Jesus, no século XVI. O primeiro registro foi na cidade de Santos, em 1543, fundada pelo colono Braz Cubas. (COSTEIRA, 2003, p.4)

No governo de Getúlio Vargas, criaram-se os Institutos de Aposentadoria e Pensões, responsáveis pelas extensões dos direitos sociais de trabalhadores, logo após de 1945 com a expansão da inclusão de serviços nas áreas de alimentação, habitação e saúde, ocorreu um período de construções de edifício público e outros mais complexos, tendo a características da arquitetura moderna brasileira (COSTEIRA, 2003, p.5). Em destaques em projetos hospitalares foram: a Maternidade Universitária de São Paulo, em 1944, de Rino Levi, o Hospital de Clínicas de Porto Alegre, em 1955, de Jorge Machado Moreira e o Hospital Sul América, atualmente com o nome Hospital da Lagoa, em 1952, de Oscar Niemeyer.

\section{PRIMEIROS HOSPITAIS ONCOLÓGICOS DO BRASIL}

Durante o governo de Getúlio Vargas, entre 1930 e 1945, aconteceram movimentos para o combate ao câncer e atender as necessidades da população. O intuito de controlar a doença construiu-se o Hospital Estácio de Sá em 1936, com serviços que contaria com 40 leitos, juntamente com ambulatórios, radioterapia e salas para cirurgias, com o término das obras em 1937. O hospital foi inaugurado em 14 de maio de 1938, na qual foi registrado o primeiro registro hospitalar de câncer do Brasil, conforme o Instituto Nacional de Câncer (INCA). Conforme relata Teixeira e Fonseca (2007, p.64)

Em 1983 inaugurou-se o CEMO (Centro Nacional de Transplante de Medula Óssea), um marco importante na área da oncologia, que permitiu aos pacientes oncológicos o acesso a tratamento adequado para seu problema de saúde no Brasil, pois na época só era possível no exterior. O projeto de expandir a assistência oncológica no Brasil (EXPANDE), criado em 1998 teve o objetivo de implantar vinte centros de alta complexidade em oncologia (CACON), que 
seriam unidades públicas ou filantrópicas que davam assistência ao paciente oncológico, com o intuito de aumentar a capacidade de atendimento integral dos serviços do SUS1.

\section{SITUAÇÃO ATUAL DA REDE DE TRATAMENTO DE CANCER}

O Instituto Nacional de Câncer José Alencar Gomes da Silva (INCA) tem o papel importante na política para prevenção e o controle do câncer no Brasil. Logo a baixo, uma definição melhor do que se trata o instituto:

O Instituto Nacional de Câncer (INCA) é o órgão auxiliar do Ministério da Saúde no desenvolvimento e coordenação das ações integradas para a prevenção e o controle do câncer no Brasil. Essas ações compreendem a assistência médico-hospitalar, prestada direta e gratuitamente aos pacientes com câncer como parte dos serviços oferecidos pelo Sistema Único de Saúde, e a atuação em áreas estratégicas, como prevenção e detecção precoce, formação de profissionais especializados, desenvolvimento da pesquisa e geração de informação epidemiológica.

E através do INCA que podemos saber também a magnitude que o câncer afeta nossa saúde pública e total de morte acarretado por essas doenças, que junto com o ministério público de saúde tentam diminuir os casos de câncer em nosso país.

As informações abaixo foram extraídas através dos dados e estudos fornecidos pelo INCA e pelo Ministério Público de Saúde em seus respectivos documentos e websites.

\section{ESTIMATIVAS DE NOVOS CASOS}

Através de estudos do INCA, estimasse que mais de 20 milhões de pessoas possuam câncer em todo mundo e no Brasil mais de 600 mil novos casos registrados todo o ano. Na qual $60 \%$ dos casos são diagnosticados já em estado avançado. E para o ano de 2016, estimasse mais de 420,310 mil novos casos, como demonstra a figura abaixo.

Figura 1 - Estimativa do número de casos novos de câncer (exceto pele não melanoma) para o ano de 2016, homens e mulheres, Brasil.

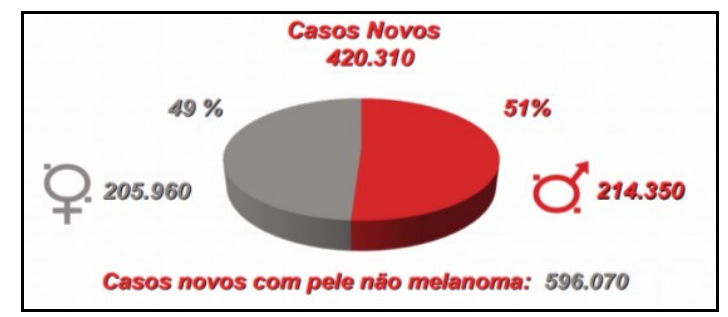

Fonte: MS / INCA / Estimativa de Câncer no Brasil, 2016

MS / INCA / Coordenação de Prevenção e Vigilância / Divisão de Vigilância

\footnotetext{
${ }^{1}$ SUS: Sistema único de saúde.
} 
E para o ano de 2030, que no mundo poderá ter mais de 27 milhões de novos casos de câncer2.

\section{REDES REGIONAIS DE ATENÇÃO À SAÚDE (RRAS)}

A implantação do RRAS foi criado em 2007 com o intuito de melhorar a eficácia da rede de Sistema Único de Saúde, o SUS, para garantir que a população tenha um acesso ao serviço de saúde público com qualidade. Para uma melhor definição, o Ministério da saúde através da Portaria GM/MS no 4279/10 as RRAS:

São definidas como arranjos organizativos de ações e serviços de saúde, de diferentes densidades tecnológicas que, integradas por meio de sistemas de apoio técnico, logístico e de gestão, buscam garantir a integralidade do cuidado em um determinado território. São caracterizadas pela formação de relações horizontais organizadas, sistematizadas e reguladas entre a atenção básica e os demais pontos de atenção do sistema de saúde.

No estado de São Paulo são divididas em 17 RRAS desde 2012, Araçatuba pertence à RRA 12, a qual possuí uma população de 2.22.578 habitantes (IBGE 2012) com uma extensão territorial de 37.604,723 km (SEADE), pertencente ao lado noroeste do Estado de São Paulo, constituída pelos departamentos regionais de Saúde: DRS II Araçatuba e DRS XV São José do Rio Preto.

O objetivo do projeto é atender a região do DRS II Araçatuba, que é constituído por três (3) regiões denominadas: dos Lagos, Central e dos Consórcios, na qual abrange quarenta (40) municípios, com uma população total de 728.743 habitantes (IBGE 2012), com porcentagem de $32,77 \%$ da população total da RRAS 12 . Conforme tabela abaixo:

Tabela 1 - Divisão político administrativa da RRAS, por municípios e população. (IBGE, 2013)

\begin{tabular}{|c|c|c|c|c|c|}
\hline \multirow{2}{*}{ RRAS } & \multirow{2}{*}{ DRS Nome } & Região de Saúde & Qtde. Municípios & População Total 2012 & \% população total \\
\hline \multirow{3}{*}{ RRAS 12 } & \multirow{3}{*}{$\begin{array}{c}\text { DRS II } \\
\text { ARAÇATUBA }\end{array}$} & Central & 11 & 281.738 & 12,61 \\
\cline { 3 - 6 } & & Dos Lagos & 12 & 192.212 & 8,60 \\
\cline { 3 - 6 } & & Dos Consórcios & 17 & 254.793 & 11,41 \\
\cline { 3 - 6 } & & Subtotal & 40 & 728.743 & 32,62 \\
\hline
\end{tabular}

Fonte: SES/SP

\section{HOSPITAIS ESPECIALIZADOS}

Para uma melhor atenção a rede de saúde oncológico, é constituída por vários tipos de serviços como: atenção ambulatorial, hospitalar e uma rede de urgência e emergência. Os hospitais especializados são classificados em UNACON E CACON, na qual suas definições seguem a baixo conforme Portaria no 874, de 16 de Maio de 2013:

\footnotetext{
${ }^{2}$ Boyle P, Le'vin B, editors. World cancer report 2008. Lyon: IARC Press; 2008.510p.
} 
UNACON: São estruturas hospitalares na qual realizam o diagnóstico definitivo e o tratamento dos cânceres mais prevalentes da região de saúde onde está inserido. Na qual seu dever é oferecer minimamente tratamentos para cirurgia e quimioterapia e obrigatoriamente tratamento radioterápico.

CACON: Realizam o diagnóstico definitivo e o tratamento de todos os tipos de câncer, mas não obrigatoriamente dos cânceres raros e infantis. Tendo obrigação de oferecer tratamento para cirurgias, radioterapia e quimioterapia dentro de sua própria estrutura hospitalar.

Logo a baixo os hospitais especializados de toda a RRAS 12.

\section{CENTRO DE TERAPIA ONCOLÓGICA DE ARAÇATUBA}

O centro de terapia oncológica (СТO) existentes na cidade de Araçatuba se localiza ao lado da Santa Casa de Misericórdia da cidade, que iniciou suas atividades em setembro de 2007. Atualmente está qualificado para tratamento de vários tipos de câncer, classificado como UNACON, com serviço de hematologia3, quimioterapia4 e radioterapia5.

Através de o registro hospitalar de câncer em 2010, o CTO de Araçatuba recebeu uma classificação de "BOM", que significa que "não apresentaram atraso no cadastro de admissões e o percentual de seguimento encontra-se acima da média do Estado" (FOSP Fundação Oncocentro de São Paulo). Porém com o passar dos anos, os atendimentos aumentaram e com isso a fila de procura por atendimento aumentou.

Nas tabelas ${ }^{6}$ abaixo (tabelas 2, 3 e 4), mostram a quantidade de tratamento oferecido pelo СTO de Araçatuba, divididos em suas respectivas atividades e regiões.

Tabela 2 - Pactuação7 em Quimioterapia - DRSII

\begin{tabular}{|c|c|c|c|}
\hline RS & Necessidade & Realizado 2013 & Serviço \\
\hline Central & 2986 & 4258 & Santa Casa de Misericórdia de Araçatuba \\
\hline Consórcios & 2701 & 1312 & Santa Casa de Misericórdia de Araçatuba \\
\hline Lagos & 2037 & 568 & Santa Casa de Misericórdia de Araçatuba \\
\hline Total & 7724 & 6.138 & \\
\hline
\end{tabular}

Fonte: Plano de ação regional de prevenção e controle de câncer.

\footnotetext{
${ }^{3}$ Hematologia: A Hematologia e a Hemoterapia são as especialidades médicas que estudam e tratam as doenças do sangue e de órgãos hematopoéticos, onde se formam as células do sangue.

4 Quimioterapia: É um tipo de tratamento médico que introduz na circulação sanguíneo composto químico, chamada quimioterápica, para combater o câncer. A medicação pode ser administrada das seguintes formas: Via oral (pela boca), Intravenosa (pela veia), Intramuscular (pelo músculo), Subcutânea (abaixo da pele), Intratecal (pela espinha dorsal) e Tópico (sobre a pele).

5 Radioterapia: A radioterapia é um tratamento no qual se utilizam radiações ionizantes ( raio-X, por exemplo), um tipo de energia direcionada, para destruir ou impedir que as células do tumor aumentem. Essas radiações não são visíveis e durante a aplicação os pacientes não sente nada.

6 Todas as tabelas foram feitas por Oncologia RRAS 12 e revisado em 03/07/2015.

7 Pactuação: A Programação Pactuada e Integrada é um processo instituído no âmbito do Sistema Único de Saúde onde, em consonância com o processo de planejamento, são definidas e quantificadas as ações de saúde para população residente em cada território, bem como efetuados os pactos Inter gestores para garantia de acesso da população aos serviços de saúde.
} 


Gerenciamento de Cidades
National Journal of Cities Management
\begin{tabular}{l|c|c|c|c|}
\hline RS & Necessidade & Realizado 2013 & Serviço \\
\hline Central & 24229 & 11482 & Santa Casa de Misericórdia de Araçatuba \\
\hline Consórcios & 21912 & 6384 & Santa Casa de Misericórdia de Araçatuba \\
\hline Lagos & 16530 & 2655 & Santa Casa de Misericórdia de Araçatuba \\
\hline Total & 62671 & 20521 & Tabela - Pactuaça em Radioterapia - DRSII \\
\hline
\end{tabular}

Fonte: Plano de ação regional de prevenção e controle de câncer.

Tabela 4 - Pactuação em Internação e Cirurgia - DRSII

\begin{tabular}{|c|c|c|c|}
\hline RS & Necessidade & Realizado 2013 & Serviço \\
\hline Central & 366 & 34 & Santa Casa de Misericórdia de Araçatuba \\
\hline Consórcios & 331 & 4 & Santa Casa de Misericórdia de Araçatuba \\
\hline Lagos & 250 & 1 & Santa Casa de Misericórdia de Araçatuba \\
\hline Total & 947 & 39 & \\
\hline
\end{tabular}

Fonte: Plano de ação regional de prevenção e controle de câncer.

\section{Públicos Alvos do CTO de Araçatuba}

O alvo de público da unidade atende todas as idades, desde criança até ao idoso. Na qual o tratamento é diferenciado para cada idade do paciente. Segue a baixo as definições de cada idade conforme a lei:

A lei no 8.069, de 13 de julho de 1990 do Estatuto da Criança e do Adolescente determina:

Art. 2o Considera-se criança, para os efeitos desta Lei, a pessoa até doze anos de idade incompletos, e adolescentes aquela entre doze e dezoito anos de idade. Parágrafo único. Nos casos expressos em lei, aplica-se excepcionalmente este Estatuto às pessoas entre dezoito e vinte e um ano de idade.

A lei $n$ ㅇ 10.741, de 1으 de outubro de 2003 do Estatuto do Idoso determina:

Art. 10 É instituído o Estatuto do Idoso, destinado a regular os direitos assegurados às pessoas com idade igual ou superior a 60 (sessenta) anos.

Em caso de tratamento de câncer, cada idade tem uma diferença crucial em seu atendimento como explica a pediatra Emma Chen Sasse:

Não podemos nos esquecer de que estamos tratando pessoas em fase de crescimento e desenvolvimento. A radioterapia e mesmo o uso de várias drogas tóxicas ao organismo podem levar, em longo prazo, a consequências desastrosas para o futuro da criança como baixa altura ou até mesmo um segundo câncer. Isto 
torna ainda mais desafiador à luta contra o câncer e estimula a procura de novos métodos de tratamento mais eficientes e menos agressivos.

\section{CONCLUSÃO}

Como podemos verificar atual situação da cidade de Araçatuba, polo regional de tratamento de câncer, apesar de ter um sistema de tratamento qualificado como "BOM", não é capaz de atender o numero de pacientes existentes na rede que precisam de cuidados. Ora pela estrutura física incapaz de ampliar leitos, ora pela falta de pactuação de serviços com o estado. Os prejuízos deste sistema incompleto podem atingir de forma dramática estes pacientes, os quais muitas vezes precisam se deslocar por grandes distancias para se tratarem em outra região, expondo-se desta maneira a viagens cansativas, estadias onerosas, e dores durante o processo de locomoção distanciado.

Verifica-se, portanto a necessidade da ampliação destes serviços, sem se esquecer da qualidade arquitetônica do espaço, afinal o objetivo da arquitetura atualmente, não é somente disponibilizar um espaço acessível, mas se preocupar com a saúde física e psicológica do paciente, proporcionando um ambiente humanizado.

\section{AGRADECIMENTO}

Agradeço primeiramente a Deus, aos meus professores, a minha orientadora Gislaine Bianchi e amigos, que me ajudaram nessa primeira etapa deste trabalho de conclusão de curso. E principalmente, dedico todo esse trabalho a minha mãe, Nair Aparecida Zorzenon Maia e a todas as pessoas que passam por algum tratamento oncológico.

\section{REFERÊNCIAS}

CCC. Centro de combate ao câncer. Disponível em:< www.cccancer.net>. Acesso em: 30 mar. 2016.

COSTEIRA, Elza Maria Alves. Arquitetura hospitalar: História, evolução e novas visões. 2003. 08 folhas. Resumo (Disertação de mestrado em Arquitetura) - Arquitetura e Urbanismo da Universidade Federal do Rio de Janeiro, Rio de Janeiro, 2003. Disponível em: <www.epublicacoes.uerj.br/index.php/sustinere/index>. Acesso em: 07 mai. 2016.

COSTI, Marilice. A influência da luz e da cor em salas de espera e corredores hospitalares. 1 ed. Porto Alegre: Editora EDIPUCRS, 2002.

FOUCAULT, Michel. Microfísica do Poder. 28 ed. Rio de janeiro: Editora Graal, 1989.

LUCKIANTCHUKI, Marieli Azoia; CARAM, Rosana Maria. Arquitetura Hospitalar e o Conforto Ambiental: Evolução Histórica e Importância na Atualidade. 2011.08 folhas. Artigo 
(Arquitetura e Urbanismo) - USP, São Carlos,2011. Disponível em: < http://www.usp.br/nutau/CD/trabalhos.html\#cas>. Acesso em: 15 abr. 2016.

MIQUELIN, Lauro Carlos. Anatomia dos edifícios hospitalares. 1 ed. São Paulo: Editora CEDAS , 1992.

ORGANIZAÇÃO MUNDIAL DE SAÚDE - Conferência de Alma Ata, 1978

PORTAL DA SAÚDE. As redes de atenção à saúde. Disponível em: <dab.saude.gov.br/portaldab/smp_ras.php>. Acesso em: 15 mar. 2016.

SANTA CASA DE ARAÇATUBA. Unidade de oncologia. Disponível em: <santacasadearacatuba.com.br/santacasa/novo/santacasa/santacasa_instalacoes_detalhes.as p?id_item=24\&categoria=Hospitalares7>. Acesso em: 25 mar. 2016.

SECRETARIA DEESTAD DA SAÚDE. Programação Pactuada e Integrada. Disponível

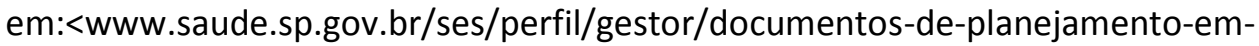
saude/programacao-pactuada-e-integrada>. Acesso em: 25 mar. 2016.

SES SP. Termo de Referência para a estruturação de Redes Regionais de Atenção à Saúde no Estado de São Paulo. Agosto de 2011, p.14. Disponível em: $<$ http://www.saude.sp.gov.br/resources/ses/perfil/gestor/homepage/redes-regionais-deatencao-a-saude-no-estado-de-sao-paulo/redes-regionais-de-atencao-a-sauderras/termo_de_referencia_redes_regionais.pdf>. Acesso em: 19 mar. 2016.

TEIXEIRA, Luiz Antônio; FONSECA, Cristina M. O. De Doença desconhecida a problema de saúde pública: o INCA e o controle do Câncer no Brasil. Rio de Janeiro: Ministério da Saúde, 2007. 172 p. Disponível em: <http://bvsms.saude.gov.br/bvs/publicacoes/doenca_desconhecida_saude_publica.pdf $>$. Acesso em: 20 mai.2016. 